\title{
Learning Commons en bibliotecas universitarias
}

\section{Una revisión dedicada a las características y desafíos de un espacio físico transformado en ambiente para el aprendizaje}

\section{Larisa González Martínez \\ Departamento de Historia, Facultad de Filosofía, Letras e Historia, División de Ciencias Sociales y Humanidades, Campus Gua- najuato, Universidad de Guanajuato, México I larisa.gonzalez.martinez@hotmail.com / http://orcid.org/oooo-0003-1364-0990}

\author{
Felipe de Jesús Jasso Peña \\ Instituto Tecnológico y de Estudios Superiores de Monterrey. Campus Monterrey | fjasso@tec.mx / \\ http://orcid.org/oooo-0002-8148-8399
}

\begin{abstract}
Resumen
El propósito de este artículo es hacer una revisión de la literatura sobre el término Learning Commons (LC) para bibliotecas universitarias, un modelo que ha pasado de una conceptualización meramente física (centrada en la transformación material de los espacios para facilitar el trabajo colaborativo y el confort), a un enfoque más académico y complejo, que contempla incluso la creación y operación de entornos virtuales, un perfil y trabajo del bibliotecario más orientado a lo académico, además de iniciativas pedagógicas de diversa índole. Así pues, se presenta una definición de este esquema bibliotecario acorde a las últimas tendencias existentes sobre el tema, enumerando sus atributos más importantes y, finalmente, se expone una breve nota sobre los retos que implica la puesta en marcha de un espacio con estas características.
\end{abstract}

Learning Commons in university libraries: A review dedicated to the characteristics and challenges of a physical space transformed into an environment for learning

\footnotetext{
Abstract

The purpose of this article is to review the literature on the term Learning Commons (LC) for academic libraries, a model that has gone from a purely physical conceptualization (focused on the material transformation of spaces to facilitate collaborative work and comfort), to a more academic and complex approach, which includes even the creation and operation of virtual environments, a librarian profile and work more academically oriented, as well as pedagogical initiatives of various kinds. Thus,

Palabras clave

Ambientes para el aprendizaje Ambientes virtuales para el aprendizaje

Diseño de Bibliotecas

Proceso de enseñanzaaprendizaje

Gestión del Conocimiento

\section{Keywords}

Learning Commons Virtual Learning Commons Library Design

Teaching-Learning Process Knowledge Management 
a definition of this library scheme is presented according to the latest trends on the subject, listing its most important attributes and, finally, it includes a brief note about the challenges involved in setting up a space like this.

Artículo recibido: 3-08-2019. Aceptado: 18-10-2019.

\section{Introducción}

En años recientes, diversas bibliotecas universitarias de todo el planeta (especialmente en el mundo anglosajón) han transformado sus estructuras para implementar el esquema bibliotecario denominado: Learning Commons o Ambientes para el aprendizaje (en adelante, LC). Estos cambios han obedecido a diversas causas, como los cambios en las maneras de aprender (más centradas en el trabajo en equipo), el aumento de las colecciones digitales en los centros de información y la disminución en la consulta de materiales impresos, entre otras cuestiones (González-Fernández-Villavicencio, 2017).

Como resultado de estas experiencias y del trabajo hecho por diversos académicos y expertos en el tema, este modelo ha cambiado, pasando de una definición basada en los espacios físicos a otra que se fundamenta en circunstancias y factores pedagógicos y educativos, que acompañan y fortalecen las dinámicas propiciadas en dichos espacios (Andrews, Wright y Raskin, 2016).

La metamorfosis en la forma de definir los LC provocó consecuencias inevitables en sus atributos más importantes y, por supuesto, en las maneras específicas en la que este modelo puede llevarse a cabo (Blummer y Kenton, 2017). Una de las más relevantes, quizá, es la propuesta de los Virtual Learning Commons o Ambientes Virtuales para el Aprendizaje (VLC), que ofrecen alternativas de trabajo interesantes para todos los miembros de una comunidad de aprendizaje (Santos, Ali y Hill, 2016). Sin embargo, los cambios abren la puerta a considerables desafíos que las bibliotecas y sus operarios deben contemplar al momento de implementar un proyecto orientado al aprendizaje.

Considerando lo anterior, este trabajo pretende presentar al lector una definición de LC más moderna, acorde con las discusiones contemporáneas sobre el tema, para lo cual se describen y enumeran sus características más importantes. A su vez, se realiza un breve análisis sobre los retos que conlleva esta forma de trabajo en bibliotecas, haciendo énfasis en la problemática de los presupuestos, la disciplina en un recinto con estos atributos y las modificaciones necesarias en el perfil del bibliotecario a fin de adecuarse a este tipo de espacios.

\section{Del espacio al ambiente: hacia una necesaria definición de los Learning Commons en bibliotecas universitarias}

Los LC irrumpieron en el panorama de las bibliotecas universitarias a finales del siglo XX y principios del siglo XXI, renovando muchos conceptos y esquemas del diseño de este tipo de recintos. Para muestra basta mencionar que existen referencias sobre este concepto de biblioteca en la literatura especializada que datan de los años 2003 y 2005 las cuales, a su vez, aluden a los primeros proyectos de LC llevados a cabo en instituciones universitarias a finales de los años noventa, concretamente en 1999 (Martínez Santos, 2016). 
Y es que, ciertamente, las propuestas de este paradigma bibliotecario resultaban innovadoras por el confort de sus espacios, en los que predominaban la luz natural, un mobiliario cómodo y agradable (de preferencia móvil), áreas destinadas al descanso y la convivencia (en las que los jardines y las cafeterías no eran para nada descartables), zonas para el trabajo en equipo y otros elementos no menos interesantes (Allison et al., 2019).

No obstante, asumir que la esencia de un LC radica en sus componentes físicos es una noción muy limitada. De hecho, hay que tener claro que, más que espacios, los LC son ambientes para el aprendizaje. Esto implica que su concepción y puesta en marcha va más allá de una mera cuestión estética, transformándose en un paradigma disruptivo por las modificaciones estructurales que provoca (Gallo-León, 2018). Para entender mejor esta idea es importante dar una definición de este esquema bibliotecario que, de acuerdo con Loertscher puede entenderse como un "ambiente de aprendizaje físico y virtual, en el que el aprendizaje de los estudiantes ocurre de forma independiente y en grupo a través de la exploración, la experimentación y la innovación"1 (Loertscher, 2011 citado por Kohout y Gavigan, 2015:18).

Por lo tanto, adaptando lo señalado por Loertscher, Koechlin y Zwaan (2011) y Moreillon (2017), para que una biblioteca sea considerada un Ambiente para el aprendizaje o Learning Commons debe tener los siguientes atributos:

\section{Espacio físico}

Este debe caracterizarse por la flexibilidad, el dinamismo y la respuesta inmediata a diferentes necesidades, mediante el cambio en la configuración del mobiliario de la biblioteca, sin renunciar a la comodidad (Helfrich, 2014). En este artículo se considera que es importante que el centro de información se transforme en una extensión del aula a través de sus elementos físicos, que adapte sus recursos a las necesidades de todos los miembros de la comunidad de aprendizaje y que propicie la interacción social (Moreillon, 2017).

Asimismo, si los presupuestos lo permiten, este tipo de edificios (sea que se trate de un recinto completamente nuevo, o bien, de la remodelación de uno ya existente) deben buscar la sustentabilidad en aspectos como la iluminación de los espacios, los servicios de agua, la climatización y calidad del aire al interior del edificio, los materiales de construcción empleados en la biblioteca y la administración y la operación cotidianas (Carr, 2013).

Cabe señalar que, en estos momentos en que los desafíos globales dirigen la mirada hacia las construcciones verdes, los LC tienen la oportunidad de integrar jardines y otros sectores naturales (Henning, 2018). Estas áreas pueden adquirir aspectos muy tradicionales (plantas y árboles en maceta), o bien, constituir proyectos más especializados como jardines infiltrantes o verticales, aprovechables para iniciativas diversas de enseñanza-aprendizaje.

De esta manera, un LC cuya operatividad posea un enfoque "verde", ecológico o sustentable puede ser también un factor de aprendizaje importante para los miembros del centro educativo a través del ejemplo y mediante la inclusión de programas enfocados en la conciencia sobre temas como el cambio climático, exhibiciones de toda clase con temáticas medioambientales (sea que se realicen con aportaciones de la comunidad educativa o con elementos de organizaciones y actores externos) y visitas guiadas. Un aspecto que también es señalado por algunos autores como parte de un paradigma "verde", es la creación de colecciones (impresas o electrónicas) sobre temáticas relacionadas con la sustentabilidad y otros temas vinculados (Antonelli, 2008). 


\section{Espacio virtual (Virtual Learning Commons -VLC)}

Consiste en un portal que puede ser definido como "un espacio en línea participativo en el que todos en la escuela pueden trabajar, crear, construir, y compartir como una comunidad" "2 (Loertscher y Marcoux, 2015:11). Este modelo virtual busca romper un poco con la inercia en la que los centros de información son los responsables exclusivos de los contenidos que suben a la red (tutoriales, guías, portales, buscadores, etcétera), con lo cual se promueve meramente un flujo de información en una sola dirección, que va del bibliotecario al usuario (Schaffhauser, 2013).

Así, con la llegada de los Ambientes Virtuales para el Aprendizaje, se espera que todos los miembros de un centro educativo interactúen entre ellos, construyan conocimiento, se comuniquen y aprendan de una manera activa. Se sugiere utilizar algún software que permita a varios individuos editar contenidos, estableciendo grados de privacidad de acuerdo con las preferencias y necesidades de la institución educativa (Loertscher y Koechlin, 2012; Singh, 2019).

Es necesario señalar que, en este artículo, se considera que un VLC no es un sustituto de los espacios en red que son requeridos para los servicios que se ofrecen al usuario, y que son responsabilidad de la biblioteca. Por lo tanto, elementos virtuales como el catálogo o el portal, deben ser de la exclusiva administración del personal de la biblioteca, evitando confundirlos con un VLC, que es una iniciativa educativa aparte, aunque integrada al proyecto del centro de información y de la institución educativa.

\section{Presencia e integración de tecnología de punta, acompañada de ayuda y soporte para los usuarios:}

Indudablemente, la tecnología educativa ha llegado para quedarse. Esto es particularmente notorio en el ambiente universitario, en el que las tecnologías de la información y las comunicaciones han propiciado nuevas formas de elaboración del conocimiento y de trabajo colaborativo entre todos los integrantes de un centro educativo (Perurena Cancio, López Riquelme y Cabañas González, 2012). Considerando lo anterior, y partiendo de la idea de que un LC es un esquema que coloca al proceso de enseñanza-aprendizaje en un lugar preponderante, el uso que se hace de factores tecnológicos es sumamente relevante. Sin embargo, la sola presencia de tecnología en un LC no es suficiente, por lo que debe de estar acompañada de expertos y mentores en el tema, que puedan auxiliar a quien lo requiera (Moreillon, 2017). Solo de esta manera se explotará la tecnología a su máxima capacidad (Takenaka, 2017).

\section{Enfoque de equipo}

Si bien las dinámicas de trabajo de un LC ocurren al interior de la biblioteca, el liderazgo de las mismas no recae exclusivamente en los bibliotecarios. En realidad, desde el momento en que este modelo de biblioteca busca facilitar el aprendizaje integrado, el trabajo colaborativo se vuelve requisito e, incluso, se transforma en una característica esencial de un ambiente de aprendizaje a un nivel operativo, no solamente educativo y/o pedagógico. Debido a esto, el bibliotecario debe vincularse necesariamente con otros miembros de la comunidad de aprendizaje en equipos híbridos o multidisciplinarios, como los estudiantes, los profesores, los ingenieros en sistemas y expertos en tecnología, los padres de familia, los administrativos, los exalumnos, el director del centro educativo, el coordinador de la biblioteca y otros (Woo, Serenko y Chu, 2019). 


\section{Espacio de experimentación para el aprendizaje}

Un escenario deseable para el correcto trabajo en un LC radica en ofrecer el espacio y los recursos de la biblioteca a la academia, en la forma de un espacio neutral en el que nuevas ideas y experiencias de aprendizaje puedan tener lugar (Calderón Jiménez, 2014). Así, el ambiente para el aprendizaje de la biblioteca se transforma en un laboratorio para experimentar en variadas iniciativas de enseñanza-aprendizaje, incluso para fines de investigación. De esta manera, la unidad de información puede poner a disposición de la comunidad de aprendizaje sus recursos y herramientas (en especial las tecnológicas y virtuales), además de favorecer la comunicación entre sus usuarios con la implementación de diversas estrategias y medios. Asimismo, en un LC se pueden poner a prueba ideas y tecnologías novedosas, antes de implementarse en el resto de las áreas de las instituciones educativas e, incluso, en otras universidades (Loertscher, 2008; Oliveira, 2016).

\section{Proyectos y actividades}

Como ha podido observarse, la ejecución de un LCimplica el desarrollo de actividades y proyectos con rasgos educativos y pedagógicos, los cuales son la verdadera esencia del modelo. A través de dichas actividades, mediante la investigación, la solución de problemas y otros métodos, se fomenta no solo el estudio sino también el hecho de que los estudiantes se hagan responsables de su propio aprendizaje (Moreillon, 2017). En este sentido, los espacios físicos y los recursos de la biblioteca que trabaja bajo este esquema, deben fortalecer y soportar el desarrollo de dinámicas de aprendizaje entre la comunidad educativa (Bennet, 2015).

Inevitablemente, este tipo de proyectos y formas de operación y trabajo presentan retos para las bibliotecas que deciden poner en marcha un LC, como se verá en el siguiente apartado.

\section{Desafíos en la implementación de ambientes para el aprendizaje en bibliotecas universitarias}

Si bien un LC abre un horizonte de nuevas experiencias para las bibliotecas que asumen la labor de implementar este modelo, la realidad es que el cambio no siempre es sencillo e, incluso a veces, resulta problemático en varios aspectos como:

\section{La transformación física del espacio y los presupuestos disponibles}

Tal como se mencionara anteriormente, los espacios físicos (o los servicios de cafetería y los jardines) no son el fin de un Learning Commons. Sin embargo, para las bibliotecas que realizan una transformación física de sus instalaciones para la operación de este esquema o que llevan a cabo la construcción de nuevos espacios, resulta evidente la carga económica que implica una decisión en esta dirección (Lippincott y Greenwell, 2011).

El problema es especialmente grave si se considera que los centros de información constantemente están sujetos a una lucha por sus presupuestos, pero al mismo tiempo, tienen la exigencia de las autoridades universitarias para la creación de nuevos entornos para el trabajo colaborativo, a fin de atender las demandas de los usuarios, especialmente los que pertenecen a nuevas generaciones y, por tanto, están acostumbrados a nuevas formas de trabajo (Griffith y Kealty, 2018).

Una posible solución para estas problemáticas es la reutilización del mobiliario existente en la biblioteca, el cual puede ser acomodado de formas diferentes (especialmente 
en agrupaciones) para generar diversos ambientes. Se puede usar, además, muebles donados, a los cuales se les puede dar un uso innovador, muy diferente a la manera en la que eran usados. En este punto hay que decir que, si bien el espacio no debe ser el factor principal en función del cual se tomen las decisiones para la elaboración de un LC (pues las cuestiones académicas y pedagógicas son más relevantes), sí puede ser un aspecto a considerar para llevar a cabo labores de descarte de la colección que abran nuevos lugares aprovechables en la biblioteca (Woo, Serenko y Chu, 2019).

Aunque es necesario mencionar, una vez más, que la búsqueda de espacios no debe precipitar la creación de directrices que afecten los materiales consultables de la biblioteca pues, aún con la llegada de los LC (cuyo enfoque es predominantemente educativo), las bibliotecas tienen el deber y el compromiso de no descuidar sus servicios de información. Así pues, para evitar la eliminación de material valioso, es recomendable analizar nuevas maneras de acomodar las colecciones impresas, que pueden ayudar incluso a delimitar los LC mediante una colocación ingeniosa de las estanterías (Oliveira, 2018).

Si el presupuesto de la biblioteca lo permite, se pueden adquirir algunos objetos que faciliten la creación de un LC, sin que esto implique la renovación absoluta de los muebles o del edificio. Un ejemplo de esto sería el uso de pizarrones, o bien elementos decorativos como lámparas, tapetes, cuadros y otros detalles, que pueden ser colocados en formas originales y creativas (Hussong-Christian, Gascho Rempel y Deitering, 2010).

Por otra parte, si bien el empleo de exhibiciones en bibliotecas no es algo nuevo (Adewale Ogunrombi, 1997), en este artículo se considera que una estrategia vinculada al diseño y la decoración que puede acercar la biblioteca a sus usuarios es la colocación de trabajos y proyectos artísticos de los miembros de la comunidad educativa (fotografía, pintura, etcétera) en muros móviles o mamparas, además del uso de esculturas para delimitar zonas. Incluso, la obra expuesta puede ser también de otros actores y/o instituciones sociales, a quienes se invite a exponer sus ideas y trabajos en la biblioteca.

A su vez, otro elemento que renueva espacios de manera sencilla y casi inmediata es la pintura. Por ello, se pueden teñir de color ciertos muros del recinto bibliotecario (e incluso algunos muebles) a fin de diferenciar los lugares para trabajar colaborativamente de los espacios destinados para la lectura en voz baja y el estudio individual (Blake, 2015).

Cabe señalar que estas áreas dedicadas al silencio no deben desaparecer en una biblioteca, ni siquiera con un esquema de trabajo de ambientes para el aprendizaje, pues existen actividades que requieren de un alto grado de concentración que muchas veces se logra únicamente con ayuda de silencio (McCaffrey y Breen, 2016). De hecho, estas zonas ofrecen una ventaja presupuestal, pues no necesitan un cambio de imagen dado que no son un concepto nuevo en bibliotecas, a diferencia de los LC que son un modelo diferente y que, por lo tanto, requieren adecuaciones.

Finalmente, si la decisión de la biblioteca es renovar el diseño en su totalidad, una transformación gradual puede facilitar el proceso en logística y costos. Por otra parte, es importante subrayar que los espacios también pueden ser dinámicos sin una inversión alta de dinero. Un medio para ello podría ser la designación de un lugar específico dentro de la biblioteca, cuya configuración sea flexible para satisfacer las necesidades de la comunidad educativa. Esto permitiría su uso de formas diferentes a lo largo del año escolar o del día, ya sea en eventos u otras actividades dedicadas al aprendizaje (Hussong-Christian, Gascho Rempel y Deitering, 2010). 
Para concluir con este apartado, se recomiendan dos estrategias más para implementar un LC, pese a las dificultades con el presupuesto. La primera de ellas es el reacomodo creativo de las herramientas tecnológicas de la biblioteca (como los equipos de cómputo) que, al igual que las colecciones impresas, pueden delimitar áreas de trabajo (Waskow, 2011).

La segunda implica que la biblioteca se renueve mediante un cambio en los servicios que ofrece a sus usuarios. Esto no quiere decir que los servicios de información deben dejarse de lado en aras de otros que, por su exceso y su novedad, afecten la operación básica y cotidiana de la biblioteca. Sin embargo (y dado que los ambientes para el aprendizaje tienen un marcado carácter pedagógico), se pueden implementar servicios académicos (educational services/user services/academic services) como centros de escritura y asesorías en matemática, operados por los bibliotecarios o por otros integrantes de la universidad (Ferer, 2012; Patrickson Stewart y Newman, 2017). Así pues, aunque no se cuente con un presupuesto importante para una transformación total de la biblioteca, la implementación de un LC tendrá lugar mediante las interacciones novedosas entre los espacios, los recursos y los servicios implementados (Serrano-Vicente, 2007).

\section{La disciplina interna y su necesaria regulación}

Un Learning Commons es un modelo dinámico y flexible que promueve la innovación educativa (Heitsch y Holley, 2011). A través de los ambientes para el aprendizaje que fomentan un enfoque no tradicional en el proceso de enseñanza-aprendizaje de todos los miembros de la universidad. No obstante, es importante recordar que este esquema de trabajo no tiene a la diversión como centro de su operación, además de que no es su objetivo principal.

Es necesario hacer esta aclaración ante el inevitable entusiasmo que este paradigma bibliotecario despierta entre alumnos y profesores, que bien encausado, puede ser el detonante de interesantes estrategias educativas y de trabajo. Pero debe subrayarse, que el frenesí que un LC genera puede causar desorden entre los miembros de la academia y el alumnado -que suele manifestarse en forma de indisciplina y, más a menudo, como ruido- (Pressley, 2017), especialmente cuando no se comprenden los alcances, limitaciones, propósitos, fundamentos y atributos de una biblioteca de este tipo (Halbert, 2010).

Así pues, indudablemente, los acuerdos son imprescindibles para la correcta operatividad de un ambiente para el aprendizaje, y deben estar orientados hacia el nivel de ruido aceptable, el cuidado del mobiliario, el tipo de actividades que puede realizarse, el consumo de alimentos y la disciplina general en el recinto (Laboon, 2015). Sobre el primero de estos aspectos, y como ya se dijo líneas arriba, la biblioteca tiene el deber de no centrarse exclusivamente en las dinámicas grupales y ofrecer a sus usuarios una atmósfera de trabajo en la que se puedan realizar diversos tipos de actividades, entre ellas las silenciosas - como cuando se lee, o se requiere concentración-(Rawal, 2014; Head, 2016).

Es recomendable establecer y garantizar el cumplimiento de políticas claras sobre el ruido permitido en las áreas comunes y los espacios en los cuales se debe respetar la tranquilidad de las personas que estudian y trabajan individualmente. De no hacerse de esta manera, se corre el riesgo de perder a ciertos usuarios que quizá se sientan desmotivados por el desorden imperante (Peterson, 2013) -o que posean una personalidad más introvertida y menos sociable, o bien, que estén realizando tareas que requieran del silencio- y que, a su vez, comenzarán a buscar otros espacios fuera de la biblioteca para llevar a cabo sus labores, aun en momentos tan delicados como los períodos de exámenes. 
Ciertamente, el trabajo del bibliotecario no debe limitarse a la contención sonora, especialmente cuando se trate de bibliotecas pequeñas que carezcan de personal numeroso. Asimismo, también es importante controlar (y, en el caso de los espacios para el trabajo y la lectura silenciosa e individual, contener) las distracciones de cualquier naturaleza, como la contaminación visual que puede resultar especialmente molesta para los usuarios (Aremu, Omoniy y Saka, 2015). Aunque se considera una preocupación común y añeja en todas las bibliotecas, cabe recalcar el hecho de que el bibliotecario no debe vivir inmerso en una batalla cotidiana y obsesiva contra el sonido, que le distraiga de sus labores diarias o de otras responsabilidades más relevantes.

En algunos espacios, como los jardines o las áreas de relajación de los Learning Commons que hayan sido construidos con estas características, se pueden crear atmósferas cordiales mediante el empleo de música clásica o ambiental y sonidos relajantes, como el ruido de las olas, de la lluvia, de aves y otros. Este tipo de sonidos ayudan a disimular el bullicio de quienes trabajan en equipo en otros apartados de la biblioteca, aunque también es posible motivar el uso de equipos personales y portátiles de audio, como los reproductores de $\mathrm{MP}_{3}$ en todas sus modalidades (Palin, 2014), que pueden enfocar la atención del usuario evitando que perciba el ruido de otras zonas de la biblioteca.

A su vez, y si los presupuestos lo permiten, se pueden adecuar los ambientes para el aprendizaje con materiales y diseños arquitectónicos que aminoren el ruido y los distractores visuales, además de recurrir a cubículos separados por paredes o cristales para aislar a los estudiantes que así lo deseen (Hyman, 2014; Mueller, 2015). Incluso, si el dinero para los ajustes de la biblioteca es suficiente, se puede hacer uso de la tecnología para monitorear el sonido producido por los usuarios, para después tomar acciones que solucionen el problema con sistemas más sofisticados para la disminución del sonido (Baca y Pierard, 2017).

No obstante lo anterior, la mejor respuesta para el problema del bullicio es el establecimiento de reglas claras. Es necesario volver a señalar que los bibliotecarios deben trabajar en reglamentos que marquen pautas de comportamiento deseable para los usuarios, a fin de evitar el deterioro de los muebles, los espacios y las colecciones por la presencia de comida en sitios no autorizados, o por el uso inadecuado y descuidado del mobiliario y otros elementos de los LC. Todo esto puede formar parte de un proceso de planeación más amplio que permita la creación de un LC con bases claras sustentadas en un modelo de aprendizaje concreto (Bennet, 2015). Así, para una planeación exitosa se pueden realizar estudios mediante encuestas, focus group, cuestionarios y otros sistemas de recolección de información entre los usuarios y otros miembros del centro educativo (Kincaid y Pfau, 2015).

Otra labor fundamental está en la creación y operación de campañas de conciencia (Olson, 1989; Stanwicks, 2016), a través de las cuales se responsabilice del uso del LC a la comunidad educativa. A fin de que esta medida sea exitosa, las estrategias deben ser realizadas con la colaboración de todos los integrantes de la universidad, como docentes, sociedades de alumnos, autoridades, padres de familia, administrativos, entre otros.

No hay que olvidar que las "atmósferas de aprendizaje" de un LC son socialmente construidas. Puede decirse que esto implica (al igual que con los espacios de trabajo silencioso, si bien con sus características y necesidades propias) una combinación de elementos que propicien el trabajo educativo como "la organización espacial subyacente, las estructuras de poder, las reglas sociales y la interacción" ${ }^{3}$ 
Evidentemente, los tres últimos elementos apelan a una cuestión social, que implica, en primer lugar, que la comunidad educativa debe hacer suyo el LC, para que las dinámicas de aprendizaje ocurran. Y esto debe suceder independientemente de los espacios creados mediante una arquitectura y un mobiliario idóneos, con lo que se refuerza la misión de un LC como ambiente más que como espacio.

Pero para que esto acontezca, la participación de la comunidad educativa en una toma de conciencia sobre los atributos y alcances del modelo es necesaria. Para ello se pueden emplear herramientas tradicionales de señalética y marketing bibliotecario como carteles, protectores de pantalla en equipos de cómputo, separadores y otros (Porat, 2013; Luca y Narayan, 2016), o bien, recurrir al establecimiento de compromisos por parte de todos los integrantes de la universidad.

\section{La ecléctica función del bibliotecario en el Learning Commons}

Ante las complicaciones antes descriptas en el manejo de algunas interpretaciones del deber ser del Learning Commons en algunas bibliotecas universitarias, surge la necesidad de conocer cómo ha evolucionado el papel del bibliotecario especialista para el funcionamiento de estos espacios (Kloppenborg y Lodge, 2010). Tal es el caso del profesor bibliotecario o teacher librarian, cuyas actividades van desde el diseño y aplicación de los programas de formación de usuarios (Godfree y Neilson, 2018), hasta el establecimiento de contactos de valor entre directivos, docentes y la biblioteca académica (McAdoo, 2010), tanto de manera presencial como virtual. Todo esto como una táctica para apoyar la formación en alumnos de orden superior en su primer año de ingreso, durante sus estudios y el postgrado (Fulkerson, Ariew y Jacobson, 2017; Majetic y Pellegrino, 2014).

Sin embargo, con las nuevas atribuciones que les han asignado a las bibliotecas académicas tales como: el fomento del aprendizaje, la innovación, el trabajo en equipo, el intercambio de ideas, facilitar la colaboración profesor-alumno, entre otras (Loertscher, Preddy y Derry, 2013), los requerimientos del desempeño profesional del bibliotecario se han incrementado en aspectos que, además de los correspondientes a la Bibliotecología, tradicionalmente no eran requeridos en su labor (Loertscher, 2008).

Estos incluyen la comprensión de las bases de la teoría educativa, los estilos de aprendizaje y los procesos cognitivos que intervienen en la adquisición de conocimiento, el diseño de un amplio repertorio de estrategias de formación dinámicas que abarquen diversas tecnologías de información y comunicación (Loertscher y Koechlin, 2014). Lo que significa que, si un profesor solicita apoyo para desarrollar actividades que favorezcan alguna investigación por medio de una adecuada exploración de las fuentes de información más relevantes para el objetivo de aprendizaje, el bibliotecario deberá conocer este lenguaje técnico-educativo, de tal forma que pueda comprender los alcances didácticos que se buscan de acuerdo al programa del curso que se esté apoyando en ese momento (Angel, 2016).

Una de las estrategias que se han propuesto para darle cumplimiento a esta moderna versión del teacher librarian está representada por una colaboración más directa en el proceso de enseñanza-aprendizaje: el bibliotecario incrustado, integrado, embebido o embedded librarian (Cabrera y Sambaíno, 2019). Esta labor implica, por parte del bibliotecario, el no esperar a que los estudiantes, los profesores 0 los investigadores acudan a solicitar algún tipo de apoyo a través de los canales establecidos (Shumaker, 2012). Hay una disposición constante del bibliotecario para llevar a cabo un trabajo colaborativo con ellos, sin importar el lugar donde se encuentren: el salón de clases, el laboratorio o su propio hogar (Freiburger, Martin 
y Nuñez, 2016). Entre sus rasgos principales destacan: el proporcionar y mantener una continua proximidad con profesores y alumnos; solicitar su participación como cotutor en los cursos regulares; conocer y adaptarse a la disciplina de las materias o investigaciones en donde vaya a trabajar; integrarse a grupos de investigación (Reale, 2015; Tumbleson, Burke y Long, 2018; Zanin-Yost, 2018).

Ahora bien, otro de los aspectos en donde habría que tomar en consideración la participación del teacher librarian y en el cual no es frecuente que sea consultado, tiene que ver con las áreas de funcionamiento y distribución de espacios en el LC, en el diseño de reglas de comportamiento, en las dinámicas de aprendizaje relacionando las colecciones bibliográficas y en la toma decisiones en cuanto a las presentes y futuras adecuaciones que se elaboren para mejorar la calidad en el servicio (McKay, 2014). La sugerencia surge a raíz de que, aunque los diseñadores y administrativos del edificio que le da albergue al LC tengan las mejores intenciones, no es posible sustituir la experiencia que los bibliotecarios poseen en referencia a la atención a usuarios (Jones y Grote, 2018).

De hecho, en distintos proyectos universitarios donde se buscó desarrollar un LC por primera vez en sus respectivas bibliotecas, dieron por sentado la colaboración directa del personal para alcanzar un aprovechamiento óptimo de las instalaciones (Thomas et al., 2015). En otros se discutió, inclusive, las ventajas y desventajas que para el fomento del aprendizaje tenía un Learning Commons y un Information Commons, entendiendo por este último un grupo de puntos de acceso de red y herramientas asociadas a la tecnología de información, situados en el contexto del estudiante y no ubicados en un solo espacio en particular; esto abarca los recursos físicos, digitales, humanos y sociales, organizados para apoyar su aprendizaje (Sheikh, 2015). La descripción anterior es interesante puesto que, cronológicamente hablando, el Information Commons, que se consolida en los años 90 con el exponencial desarrollo tecnológico, es el antecesor directo del Learning Commons, pero la discusión en cuanto al uso adecuado de los espacios de la biblioteca académica y la forma de administrarlos día a día continúa hasta nuestros días (Little, 2014).

Finalmente, el objetivo de un bibliotecario académico sigue siendo el mismo: promover el aprendizaje entre los estudiantes. Lo único que se pretende dejar en claro es el valor de las aportaciones que, desde su experiencia con la administración de las colecciones bibliográficas, la gerencia de los servicios y el desarrollo de las competencias relacionadas al proceso educativo, puedan aportar en el diseño, implementación y aprovechamiento de los espacios, pero sobre todo, hacer que los estudiantes acudan al Learning Commons (Lux, Snyder y Boff, 2016).

\section{Conclusiones}

Con el paso del tiempo, los Learning Commons han cambiado, convirtiéndose en un concepto más complejo que aquél que inicialmente se había desarrollado, y el cual estaba centrado en las características físicas de las bibliotecas. El foco de atención puesto en la labor pedagógica y educativa que este tipo de esquema propone, ofrece al bibliotecario una amplia gama de posibilidades para llevar a cabo su tarea. Pero, para ello, es necesario prepararse y capacitarse, además de fortalecer los vínculos con la academia y tener una participación más activa en la comunidad educativa.

La clave para la correcta implementación de un LC es la información, el conocimiento a conciencia de lo que implica el desarrollo de un modelo como este. Incluso, en el caso del bibliotecario, le ayudará a saber lo que esta forma de trabajo representa para su perfil profesional. Y es que este desconocimiento puede generar la mala 
administración de los espacios y servicios de la biblioteca, produciendo desorden y un desaprovechamiento de los recursos (materiales, económicos, tecnológicos y humanos) invertidos.

Por otra parte, el no tener una idea clara al momento de emprender la creación de un LC, puede provocar estrés en las instituciones, por la concepción errónea de que dicho proyecto implica, necesariamente, una cantidad importante de dinero para ponerse en marcha. Sin embargo, partiendo de la idea de que en un LC lo verdaderamente importante son las dinámicas educativas, se puede hacer uso de toda clase de estrategias para abaratar los costos, una cuestión que puede aligerar la carga para los bibliotecarios y las autoridades de la comunidad educativa. 


\section{Q Referencias Bibliográficas}

"Adewale Ogunrombi, Samuel. 1997. Exhibitions in university libraries. En Library Review. Vol. 46, no. 6, 428-433. <https://doi-org.e-revistas.ugto. $\mathrm{mx} / 10.1108 / 00242539710178434>$

»Allison, Deeann; Erica DeFrain; Brianna D. Hitt y David C. Tyler. 2019. Academic library as learning space and as collection: A learning commons' effects on collections and related resources and services. En The Journal of Academic Librarianship. Vol. 45 no. 3, 305-314. <https://doi.org/10.1016/j.acalib.2019.04.004>

»Andrews, Camille; Sara E. Wright y Howard Raskin. 2016. Library learning spaces: Investigating libraries and investing in student feedback. En Journal of Library Administration. Vol. 56, no. 6, 647-672. <https://doi.or g/10.1080/01930826.2015.1105556>

»Angel, Christine M. 2016. Collaboration among faculty members and community partners: Increasing the quality of online library and information science graduate programs through academic service-learning. En Journal of Library $\&$ Information Services in Distance Learning. Vol. 10, no. 1-2, 4-14. <https://doi.org/ 10.1080/1533290X.2016.1240741>

"Antonelli, Monika. 2008. The Green library movement: An overview of green library literature and actions from 1979 to the future of green libraries. En Electronic Green Journal. Vol. 1, no. 27. <https://escholarship.org/uc/item/39d3v236> [Consulta: 30 junio 2019].

»Aremu, A.S., J. O. Omoniy y T. Saka. 2015. Indoor Noise in Academic Libraries: A Case Study of University of Ilorin Main Library, Nigeria. En African Journal of Library, Archives \& Information Science. Vol. 25, no. 1, 5-14. <http://search.ebscohost.com.e-revistas.ugto. $\mathrm{mx} /$ login.aspx?direct=true $\& \mathrm{db}=\mathrm{agh} \& A \mathrm{~N}=102893878$ \&lang=es\&site=ehost-live $>$ [Consulta: 1 agosto 2019].

"Baca, Olivia y Cindy Pierard. 2017. Finding the Sonic Sweet Spot: Implementing a Noise Management Program in a Library Learning Commons. En 2017 Access Services Conference (2017: Atlanta). Trabajos presentados. Atlanta: Access Services Conference. <https://smartech.gatech.edu/bitstream/handle/1853/59404/Finding\%20the\%20Sonic\%20Sweet\%20Spot\%20Poster\%20 Baca\%20Pierard\%202017AS\%20\%20Conference.pdf? sequence $=1 \&$ is Allowed=y"> [Consulta: 20 junio 2018].

»Bennet, Scott. 2015. Putting learning into library planning. En Portal: Libraries and the Academy. Vol. 15, no. 2, 215-231. <https://doi.org/10.1353/pla.2015.0014>

»Blake, Sheila L. 2015. The challenges of creating a Learning Commons. Warrensburg, Missouri: University of Central Missouri. <https://jckldigital. ucmo.edu/bitstream/handle/123456789/415/Blake201530_RP_Challenges. pdf? sequence $=7$ \&isAllowed $=y>$ [Consulta: 19 junio 2018].

»Blummer, Barbara y Jeffrey M. Kenton. 2017. Learning commons in academic libraries: Discussing themes in the literature from 2001 to the present. En New Review of Academic Librarianship. Vol. 23, no. 4, 329-352. <https://doi.or g/10.108o/13614533.2017.1366925>

»Carr, Mary M. 2013. The Green Library Planner: What Every Librarian Needs to Know Before Starting to Build or Renovate. Lanham: Scarecrow Press. <http:// 
search.ebscohost.com.e-revistas.ugto.mx/login.aspx?direct=true\&db=nlebk\& AN=1449926\&lang=es\&site=ehost-live $>$ [Consulta: 30 junio 2019].

" Cabrera, Gabriela y Samira Sambaíno. 2019. Experiencias del bibliotecólogo integrado dentro de los ciclos de investigación en bibliotecas universitarias de Uruguay. En IFLA World Library and Information Congress (85를 2019: Atenas). Trabajos presentados. Atenas: IFLA. p. 1-9. <http://library.ifla.org/2577/1/o82cabrera-es.pdf>. [Consulta: 29 septiembre 2019].

»Calderón Jiménez, Andrea. 2014. Propuesta de implementación de learning commons en bibliotecas de educación superior de Costa Rica como aplicación de un servicio de calidad. Monterrey: Universidad TecVirtual. Escuela de Graduados en Educación. 137 p. Tesis de doctorado. <https://repositoriotec.tec.ac.cr/ handle/2238/9672> [Consulta: 29 septiembre 2019].

»Cox, Andrew M. 2017. The Experience of Learning Atmosphere in the Learning Commons. En iConference 2017 Proceedings. p. 333-339. <https://doi. org/10.9776/17203>

»Ferer, Elise. 2012. Working together: library and writing center collaboration. En Reference Services Review. Vol. 40, no. 4, 543-557. <https:// ugto-primo.hosted.exlibrisgroup.com/permalink/f/196kdco/TN_emerald_ S10.1108/00907321211277350> [Consulta: 31 julio 2019].

" Freiburger, Gary; Jennifer R. Martin y Annabelle V. Nuñez. 2016. An embedded librarian program: Eight years on. En Medical Reference Services Quarterly. Vol. 35, no. 4, 388-396. <https://doi.org/10.1080/02763869.2016.1220756>

»Fulkerson, Diane M.; Susan Andriette Ariew y Trudi E. Jacobson. 2017. Revisiting metacognition and metaliteracy in the ACRL framework. En Communications in Information Literacy. Vol. 11, no 1, 21-41. <https://doi.org/10.1576o/comminfolit.2017.11.1.45>

»Gallo-León, José-Pablo. 2018. Los cuatro espacios: un modelo para la organización física de la biblioteca. En Anuario ThinkEPI. Vol. 12, 104-112. <https://doi. org/10.3145/thinkepi.2018.11>

" Godfree, Holly y Olivia Neilson. 2018. School libraries matter! The missing piece in the education puzzle. En Access E-journal. Vol. 1, 28-41. <https://search. informit.com.au/documentSummary; dn=480238959244309; res=IELHSS> [Consulta: 14 noviembre 2018].

»González-Fernández-Villavicencio, Nieves. 2017. Espacios físicos de la biblioteca universitaria en el nuevo ecosistema de aprendizaje. En Anuario ThinkEPI. Vol. 11, 109-118. <https://doi.org/10.3145/thinkepi.2017.14>

" Griffith, Andrew S. y Ceire Kealty. 2018. Are academic libraries utilized to produce engaged alumni? En Library Management. Vol. 39, no. 3/4, 200-206. <https:// doi.org/10.1108/LM-09-2017-0096>

»Halbert, Martin. 2010. The information commons: A platform for innovation. En Journal of Library Administration. Vol. 50, no. 1, 67-74. <https://doi. org/10.1080/01930820903422107>

»Head, Alison J. 2016. A project information literacy research report the practitioner series. <http://www.projectinfolit.org/uploads/2/7/5/4/27541717/pil_libspace_report_no_methods_12_6_16.pdf>. [Consulta: 31 julio 2019].

»Heitsch, Elizabeth K. y Robert P. Holley. 2011. The information and learning commons: Some reflections. En New Review of Academic Librarianship. Vol. 17, no. 1, 64-77. <https://doi.org/10.1080/13614533.2011.547416> 
》Helfrich, Jennifer. 2014. Creative Spaces: Flexible Environments for the 21stCentury Learner. En Knowledge Quest. Vol. 42, no. 5, 76-77. <http://search.ebscohost.com.e-revistas.ugto. $\mathrm{mx} /$ login.aspx?direct=true $\& \mathrm{db}=\mathrm{agh} \& \mathrm{AN}=95795527$ \&lang=es\&site=ehost-live. $>$ [Consulta: 4 diciembre 2018].

" Henning, Judy. A. 2018. Proactive Advocacy: Necessary for New School Library Construction. En Teacher Librarian. Vol. 45, no. 3, 24-28. <http://search.ebsco-

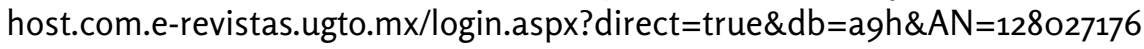
\&lang=es\&site=ehost-live $>$ [Consulta: 4 diciembre 2018].

" Hyman, Shannon C. 2014. Planning and creating a Library Learning Commons. En Teacher Librarian. Vol. 41, no. 3, 16-21. <http://search.ebscohost.com.e-revistas.ugto. $\mathrm{mx} /$ login.aspx?direct $=$ true $\& d b=a 9 h \& A N=94736155 \&$ lang $=e s \&$ site $=$ ehost-live > [Consulta: 20 junio 2018].

"Hussong-Christian, Uta; Hannah Gascho Rempel y Anne-Marie Deitering. 2010. The Library as Learning Commons: Rethink, Reuse, Recycle. En College \& Undergraduate Libraries. Vol. 17, no. 2-3, 273-286. <https://doi.or g/10.108o/10691316.2010.481951>

"Jones, Derek y Andrew Grote. 2018. The library as learning commons. En Planning for Higher Education. Vol. 46, no. 3, 1-9. <https://www.scup.org/resource/ the-library-as-learning-commons/> [Consulta: 30 julio 2019].

» Kincaid, Kami y Peter Pfau. 2015. Creating ever-evolving, school-specific learning commons. En Teacher Librarian. Vol. 42, no. 4, 8-14. <http://search.ebscohost.com.e-revistas.ugto. $\mathrm{mx} /$ login.aspx?direct=true $\& \mathrm{db}=\mathrm{agh} \& A \mathrm{~N}=102336189$ \&lang=es\&site=ehost-live > [Consulta: 22 junio 2019].

» Kloppenborg, Paul y Damian Lodge. 2010. Forgotten anything: Library staff competencies for the learning commons. En Library Management. Vol. 31, no. 3, 169-185 <https://doi.org/10.1108/01435121011027345>

"Kohout, Jessica y Karen Gavigan. 2015. The Years of Our Learning Commons. En Teacher Librarian. Vol. 43, no. 2, 18-23. <http://search.ebscohost.com.e-revistas. ugto. $\mathrm{mx} / \operatorname{login}$. aspx? direct $=$ true $\& \mathrm{db}=\mathrm{agh} \& A \mathrm{~N}=111875239 \&$ lang=es $\&$ site $=$ ehos t-live> [Consulta: 1 noviembre 2018].

»Laboon, Jennifer. 2015. Building a Learning Commons Culture: Teaching Procedures. <https://knowledgequest.aasl.org/building-a-learning-commons-culture-teaching-procedures/> [Consulta: 1 agosto 2019].

» Lippincott, Joan y Stacey Greenwell. 2011. 7 Things You Should Know About the Modern Learning Commons. En EDUCAUSE Learning Initiative (ELI). 11- Abril. $<$ https://library.educause.edu/resources/2011/4/7-things-you-should-knowabout-the-modern-learning-commons>. [Consulta: 30 agosto 2019].

» Little, Geoffrey. 2014. Space: The final frontier. En The Journal of Academic Librarianship. Vol. 40, no. 6, 632-633 <https://doi.org/10.1016/j.acalib.2014.10.006>

» Loertscher, David V. 2008. Flip This Library. En School Library Journal. Vol. 54, no. 11, 46-48. <http://search.ebscohost.com.e-revistas.ugto.mx/login.aspx?direct=tr

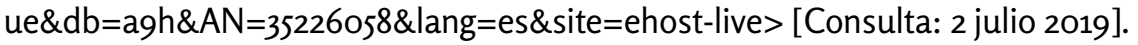

"Loertscher, David V. y Carol Koechlin. 2012. The Virtual Learning Commons and School Improvement. En Teacher Librarian. Vol. 39, no. 6, 20-24. <http:// search.ebscohost.com.e-revistas.ugto.mx/login.aspx?direct=true \&db=agh\&A $\mathrm{N}=82862806 \&$ lang=es\&site=ehost-live $>$ [Consulta: 2 noviembre 2018].

»Loertscher, David V. y Carol Koechlin. 2014. Climbing to excellence: Defining characteristics of successful learning commons. En Knowledge Quest. Vol. 42, 
no. 4, 2-11. <http://www.ala.org/aasl/sites/ala.org.aasl/files/content/aas/pubsandjournals/knowledgequest/docs/KQ_MarApr14_ClimbingtoExcellence. pdf> [Consulta: 30 noviembre 2018].

" Loertscher, David V.; Carol Koechlin y Sandi Zwaan. 2011. The new Learning Commons: Where learners win! 2a ed. Salt Lake City: Hi Willow.

"Loertscher, David y Elizabeth Marcoux. 2015. Learning Commons Progress Report. En Teacher Librarian.Vol. 42, no. 3, 8-11. <http://search.ebscohost.com.erevistas.ugto. $\mathrm{mx} /$ login.aspx direct $=$ true $\& \mathrm{db}=\mathrm{agh} \& \mathrm{AN}=101109241 \&$ lang $=e s \& \mathrm{~s}$ ite $=$ ehost-live $>$ [Consulta: 4 diciembre 2018].

»Loertscher, David V.; Leslie Preddy y Bill Derry. 2013. Makerspaces in the school library learning commons and the UTEC maker model. En Teacher Librarian. Vol. 41, no. 2, 48-51. <http://search.ebscohost.com.e-revistas.ugto.mx/login.a spx?direct $=$ true $\& \mathrm{db}=\mathrm{agh} \& \mathrm{AN}=92991119 \&$ lang=es\&site=ehost-live $>$ [Consulta: 2 julio 2019].

"Luca, Edward y Bhuva Narayan. 2016. Signage by Design: A Design-Thinking Approach to Library User Experience. En Journal of Library User Experience. Vol. 1, no. 5. <http://dx.doi.org/10.3998/weave.12535642.0001.501>

" Lux, Vera; Robert J. Snyder y Colleen Boff. 2016. Why users come to the library: A case study of library and non-library units. En The Journal of Academic Librarianship. Vol. 42, no. 2, 109-117. <https://doi.org/10.1016/j.acalib.2016.01.004>

" Majetic, Cassie y Catherine Pellegrino. 2014. When science and information literacy meet: An approach to exploring the sources of science news with nonscience majors. En College Teaching. Vol. 62, no. 3, 107-112. <https://doi.or g/10.1080/87567555.2014.916650>

» Martínez Santos, Diana Carolina. 2016. Repensar las bibliotecas de la Universidad de La Salle: una revisión conceptual para el diseño de un nuevo modelo del Sistema de Bibliotecas. En Revista de la Universidad de La Salle. No. 71, 135-165. <https://ciencia.lasalle.edu.co/ruls/vol2016/iss71/7/> [Consulta: 28 septiembre 2019].

" McCaffrey, Ciara y Michelle Breen. 2016. Quiet in the Library: An EvidenceBased Approach to Improving the Student Experience. En Portal: Libraries and the Academy. Vol. 16, no. 4, 775-791. <https://doi.org/10.1353/pla.2016.0052>

» McKay, Richard. 2014. Building a learning commons: necessary conditions for success. En Community \& Junior College Libraries. Vol. 20, no. 3-4, 107-112. <https://doi.org/10.1080/02763915.2015.1056705>

" McAdoo, Monty L. 2010. Building bridges. Connecting faculty, students, and college library. U.S.A: American Library Association.

» Moreillon, Judi. 2017. The Learning Commons. En Teacher Librarian. Vol. 44, no. 3, 21-25. <http://search.ebscohost.com.e-revistas.ugto.mx/login.aspx?direct=t

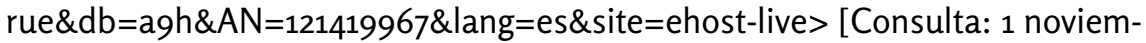
bre 2018].

" Mueller, Lisa. 2015. From Library to Learning Commons. En Teacher Librarian. Vol. 43, no. 1, 12-17. <http://search.ebscohost.com.e-revistas.ugto.mx/login.as px?direct=true $\& \mathrm{db}=\mathrm{a}$ h $\& A \mathrm{~N}=110469422 \&$ lang $=$ es \&site=ehost-live $>$ [Consulta: 22 junio 2019].

»Oliveira, Silas M. 2016. Space preference at James White Library: What students really want. En The Journal of Academic Librarianship. Vol. 42, 355-367. <http://dx.doi.org/10.1016/j.acalib.2016.05.009> 
»Oliveira, Silas M. 2018. Trends in Academic Library Space: From book boxes to Learning Commons. En Open Information Science. Vol. 2, no. 1, 59-74. <https:// doi.org/10.1515/opis-2018-0005>

»Olson, Renee. 1989. “All Quiet on the Reference Front”: Reading Public's PR Campaign, 1987-1988. En Library Journal. Vol. 114, no. 3, 140-141. <http://search. ebscohost.com.e-revistas.ugto.mx/login.aspx?direct=true\&db=agh\&AN=8904 031093\&lang=es\&site $=$ ehost-live $>$ [Consulta: 9 noviembre 2018].

"Palin, Ray. 2014. Looking for Peace and Quiet. En Knowledge Quest. Vol. 42, no. 4, 16-21. <http://search.ebscohost.com.e-revistas.ugto.mx/login.aspx?direct=t rue $\& \mathrm{db}=\mathrm{agh} \& \mathrm{AN}=94845349 \&$ lang $=$ es $\&$ site $=$ ehost-live $>$. [Consulta: 9 noviembre 2018].

»Patrickson Stewart, Sonia G y Nadine Newman. 2017. User Services in the Digital Environment. En Library Review. Vol. 66, no. 4/5, 213-234. <https://ugtoprimo.hosted.exlibrisgroup.com/permalink/f/196kdco/TN_emerald_s10.1108/ LR-07-2016-0058> [Consulta: 31 julio 2019].

"Perurena Cancio, Lilliam; Silvia López Riquelme y Jesús Cabañas González. 2012. Gestión e innovación en Tecnología Educativa: experiencias. En Economía y Desarrollo. Vol. 148, no. 2, 247-257. <http://www.redalyc.org/articulo. oa? id=425541206015> [Consulta: 3 noviembre 2018].

"Peterson, Nicole Kay. 2013. The developing role of the university library as a student learning commons: Implications to the interior spaces within. lowa: lowa State University. 128 p. Tesis de maestría. <https://doi.org/10.31274/etd-180810-3678>

" Porat, Lynne. 2013. Marketing and Assessment in Academic Libraries: A Marriage of Convenience or True Love? En Evidence based library $\&$ information practice. Vol. 8, no. 2, 60-6. <https://doi.org/10.18438/B8FS5M>

»Pressley, Lauren. 2017. Charting a Clear Course: A State of the Learning Commons. En ACRL 2017. At the helm leading transformation. (2017: Baltimore, Maryland) Trabajos presentados. Baltimore, Maryland: ACRL. p. 112-119. <http:// www.ala.org/acrl/sites/ala.org.acrl/files/content/conferences/confsandpreconfs/2017/ChartingaClearCourse.pdf> [Consulta: 22 junio 2019].

"Rawal, Jyoti. 2014. Libraries of the future: Learning Commons a case study of a State University in California. Arcata: Humboldt State University. 82 p. Tesis de Maestría. <http://humboldt-dspace.calstate.edu/bitstream/handle/10211.3/134872/ rawal_jyoti_thesis_12_10_14.pdf? sequence=1> [Consulta: 30 julio 2019].

"Reale, Michelle. 2015. Becoming an embedded librarian: Making connections in the classroom. U.S.A: American Library Association.

"Santos, leda M.; Nagla Ali y Anthony Hill. 2016. Students as Co-designers of a Virtual Learning Commons: Results of a Collaborative Action Research Study. En The Journal of Academic Librarianship. Vol. 42, no. 1, 8-14. <http://search.ebscohost.com.e-revistas.ugto.mx/login. aspx?direct $=$ true $\& d b=a g h \& A N=11197530$ 4\&lang=es\&site=ehost-live > [Consulta: 21 junio 2019].

"Schaffhauser, Dian. 2013. Will this website saveyour library (and Your Librarians)? En The Journal. Vol. 40, no. 11, 22-28. <http://search.ebscohost.com.e-revistas. ugto. $\mathrm{mx} /$ login. aspx?direct $=$ true $\& \mathrm{db}=a 9 h \& A N=94425260 \&$ lang $=$ es \&site $=e-$ host-live> [Consulta: 3 noviembre 2018].

" Serrano-Vicente, Rocío. 2007. Los Learning Centers en el Reino Unido. Estudio de caso de seis universidades medianas. En El profesional de la información. Vol. 16, no. 4, 307-318. <https://doi.org/10.3145/epi.2007.jul.o4> 
"Sheikh, Arslan. 2015. Development of information commons in university libraries of Pakistan: The current scenario. En The Journal of Academic Librarianship. Vol. 41, 130-139. <https://doi.org/10.1016/j.acalib.2015.01.002>

"Shumaker, David. 2012. Embedded librarian: Innovative strategies for taking knowledge where it's needed. U.S.A: Information Today, Inc.

"Singh, Punit Kumar. 2019. Establishing library learning commons in universities of India: A case study of BHU Library System. En Library Philosophy and Practice (e-journal), 2477. <https://digitalcommons.unl.edu/libphilprac/2477> [Consulta: 30 julio 2019].

»Stanwicks, Kabel Nathan. 2016. Zoning the Library for Silent, Quiet, and Collaborative Study. En Journal of the Library Administration EManagement Section. Vol. 12, no. 2. <https://scholarsarchive.library.albany.edu/jlams/vol12/iss2/4> [Consulta: 1 agosto 2019].

» Takenaka, Yoshikazu. 2017. A survey of the effective utilization of services provided at learning commons in Japan. En International Conference of Educational Innovation through Technology. (6a:7-9 Diciembre 2017: Osaka, Japan). Trabajos presentados. <https://doi.org/10.1109/EITT.2017.31>

» Thomas, Brittney; Sam Van Horne; Wayne Jacobson y Matthew Anson. 2015. The design and assessment of the Learning Commons at the University of lowa. En The Journal of Academic Librarianship. Vol. 41, no. 6, 804-813. <https://doi. org/10.1016/j.acalib.2015.09.005>

" Tumbleson, Beth; John Burke y Jessica Long. 2018. Assessment, analytics and analysis: Demonstrating the impact of LMS embedded librarians on student learning. En Journal of Library \& Information Services in Distance Learning. Vol. 13, no. 1-2, 196-214. <https://doi.org/10.1080/1533290X.2018.1499252>

»Waskow, Linda M. 2011. The Journey from Library Media Center to Learning Commons. En Teacher Librarian. Vol. 38, no. 5, 8-14. <http://search.ebscohost. com.e-revistas.ugto. $\mathrm{mx} /$ login.aspx?direct=true $\& \mathrm{db}=\mathrm{agh} \& \mathrm{AN}=62570020 \&$ lang $=e s \&$ site $=$ ehost-live $>$ [Consulta: 1 agosto 2019].

»Woo, Esther M.W.; Alexander Serenko y Samuel K.W. Chu. 2019. An exploratory study of the relationship between the use of the Learning Commons and students' perceived learning outcomes. En The Journal of Academic Librarianship. Vol. 45, no. 4, 413-419. <https://doi.org/10.1016/j.acalib.2019.05.007>

»Zanin-Yost, Alessia. 2018. Academic collaborations: Linking the role of the liaison/embedded librarian to teaching and learning. En College \& Undergraduate Libraries. Vol. 25, no. 2, 150-163. <https://doi.org/10.108o/10691316.2018.1455548> 
